\title{
AMBER Force Field Parameters for Cobalt-Containing Biological Systems: A Systematic Derivation Study
}

Hamed Haghshenas ${ }^{a}$, Hossein Tavakol, ${ }^{*}, b$ Bita Kavianic and Gholamhossein Mohammadnezhad ${ }^{b}$

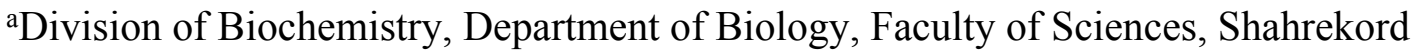
University, Shahrekord, Iran. Email: hamed.haghshenas@stu.sku.ac.ir

bDepartment of Chemistry, Isfahan University of Technology, Isfahan 84156-83111, Iran; Tell: +98-3133913241; Fax: +98-33912350; Email: $\underline{h \text { tavakol@,cc.iut.ac.ir }}$

'Division of Genetics, Department of Biology, Faculty of Sciences, Islamic Azad University, Shahrekord Branch, Shahrekord, Iran. Email: b.kaviani@std.iaushk.ac.ir 
Table S1. The detailed structure and all bonding parameters of structure C1

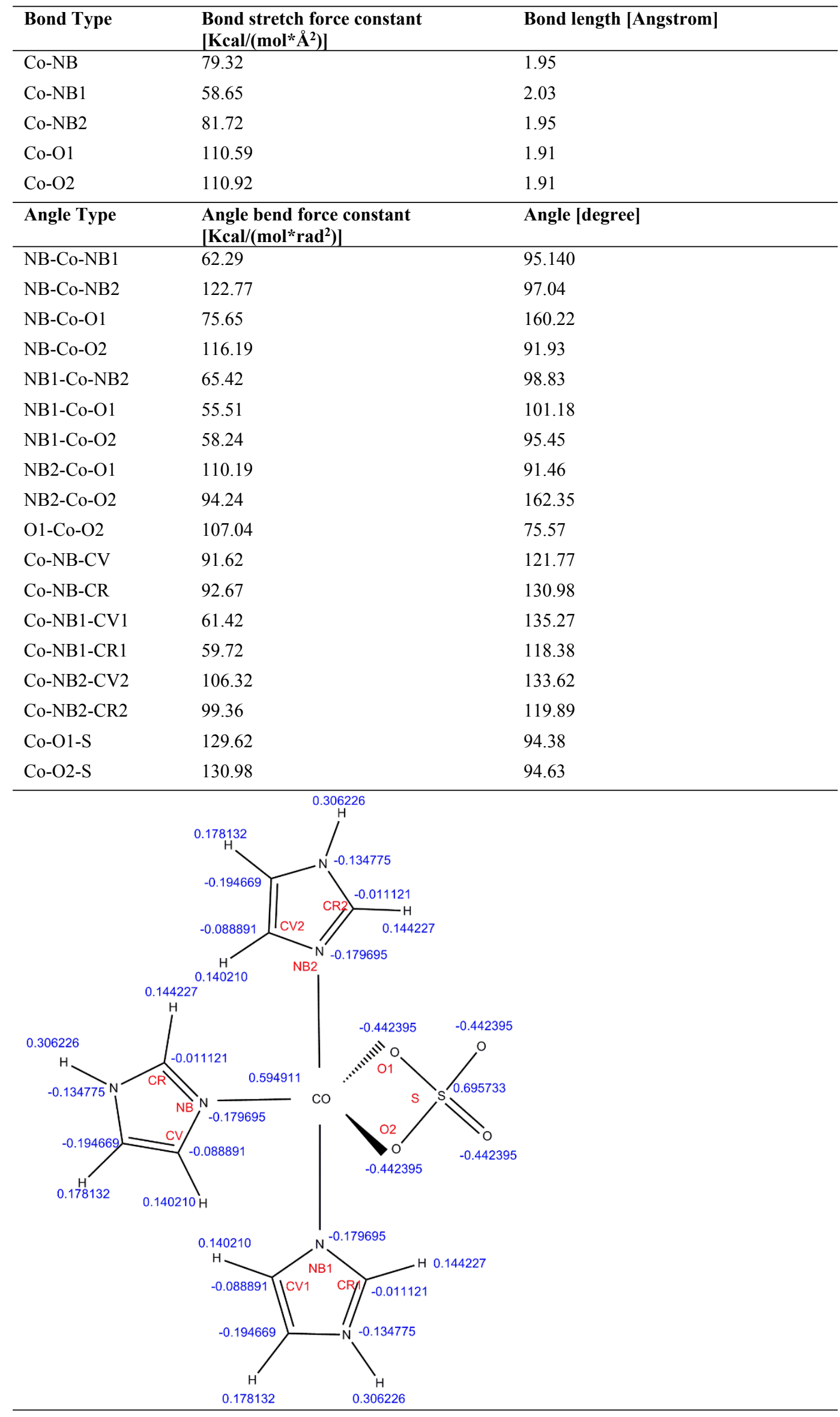


Table S2. The detailed structure and all bonding parameters of structure C2

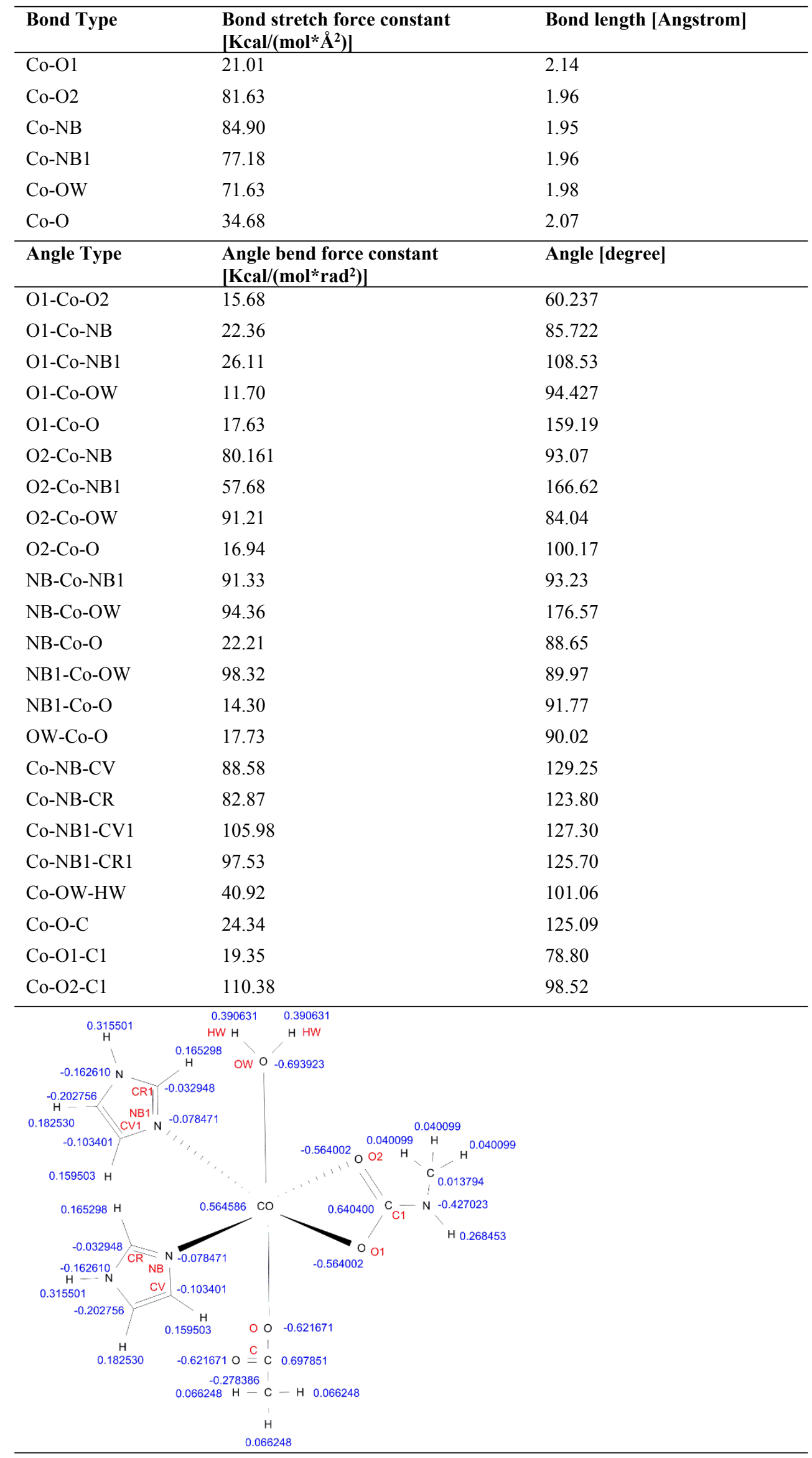


Table S3. The detailed structure and all bonding parameters of structure C3

\begin{tabular}{|c|c|c|}
\hline Bond Type & $\begin{array}{l}\text { Bond stretch force constant } \\
{\left[\mathrm{Kcal} /\left(\mathrm{mol}^{*} \AA^{2}\right)\right]}\end{array}$ & Bond length [Angstrom] \\
\hline $\mathrm{Co}-\mathrm{O}$ & 74.68 & 1.93 \\
\hline $\mathrm{Co}-\mathrm{O} 1$ & 119.40 & 1.88 \\
\hline $\mathrm{Co}-\mathrm{O} 2$ & 113.00 & 1.89 \\
\hline $\mathrm{Co}-\mathrm{O} 3$ & 96.13 & 1.93 \\
\hline Co-OW & 98.90 & 1.92 \\
\hline $\mathrm{Co}-\mathrm{ON}$ & 86.67 & 1.90 \\
\hline Angle Type & $\begin{array}{l}\text { Angle bend force constant } \\
{\left[\mathrm{Kcal} /\left(\mathrm{mol}^{*} \mathbf{r a d}^{2}\right)\right]}\end{array}$ & Angle [degree] \\
\hline $\mathrm{O}-\mathrm{Co}-\mathrm{O} 1$ & 125.70 & 172.31 \\
\hline $\mathrm{O}-\mathrm{Co}-\mathrm{O} 2$ & 74.30 & 89.03 \\
\hline $\mathrm{O}-\mathrm{Co}-\mathrm{O} 3$ & 75.01 & 88.33 \\
\hline O-Co-OW & 87.53 & 93.64 \\
\hline $\mathrm{O}-\mathrm{Co}-\mathrm{ON}$ & 106.99 & 82.85 \\
\hline $\mathrm{O} 1-\mathrm{Co}-\mathrm{O} 2$ & 116.83 & 93.42 \\
\hline $\mathrm{O} 1-\mathrm{Co}-\mathrm{O} 3$ & 118.93 & 88.41 \\
\hline O1-Co-OW & 88.00 & 93.41 \\
\hline O1-Co-ON & 116.09 & 90.02 \\
\hline $\mathrm{O} 2-\mathrm{Co}-\mathrm{O} 3$ & 111.99 & 173.13 \\
\hline $\mathrm{O} 2-\mathrm{Co}-\mathrm{OW}$ & 118.42 & 94.91 \\
\hline $\mathrm{O} 2-\mathrm{Co}-\mathrm{ON}$ & 85.10 & 86.57 \\
\hline O3-Co-OW & 113.55 & 91.57 \\
\hline O3-Co-ON & 84.05 & 86.81 \\
\hline OW-Co-ON & 109.06 & 176.18 \\
\hline Co-O-C & 94.80 & 108.34 \\
\hline $\mathrm{Co}-\mathrm{O} 1-\mathrm{C} 1$ & 126.14 & 126.25 \\
\hline $\mathrm{Co}-\mathrm{O} 2-\mathrm{C} 2$ & 116.30 & 124.34 \\
\hline $\mathrm{Co}-\mathrm{O} 3-\mathrm{C} 3$ & 119.02 & 125.40 \\
\hline Co-OW-HW & 56.61 & 102.49 \\
\hline Co-ON-N & 116.90 & 114.23 \\
\hline (1) & $=\left.\right|_{C} ^{-0.278772}$ & \\
\hline
\end{tabular}


Table S4. The detailed structure and all bonding parameters of structure C4

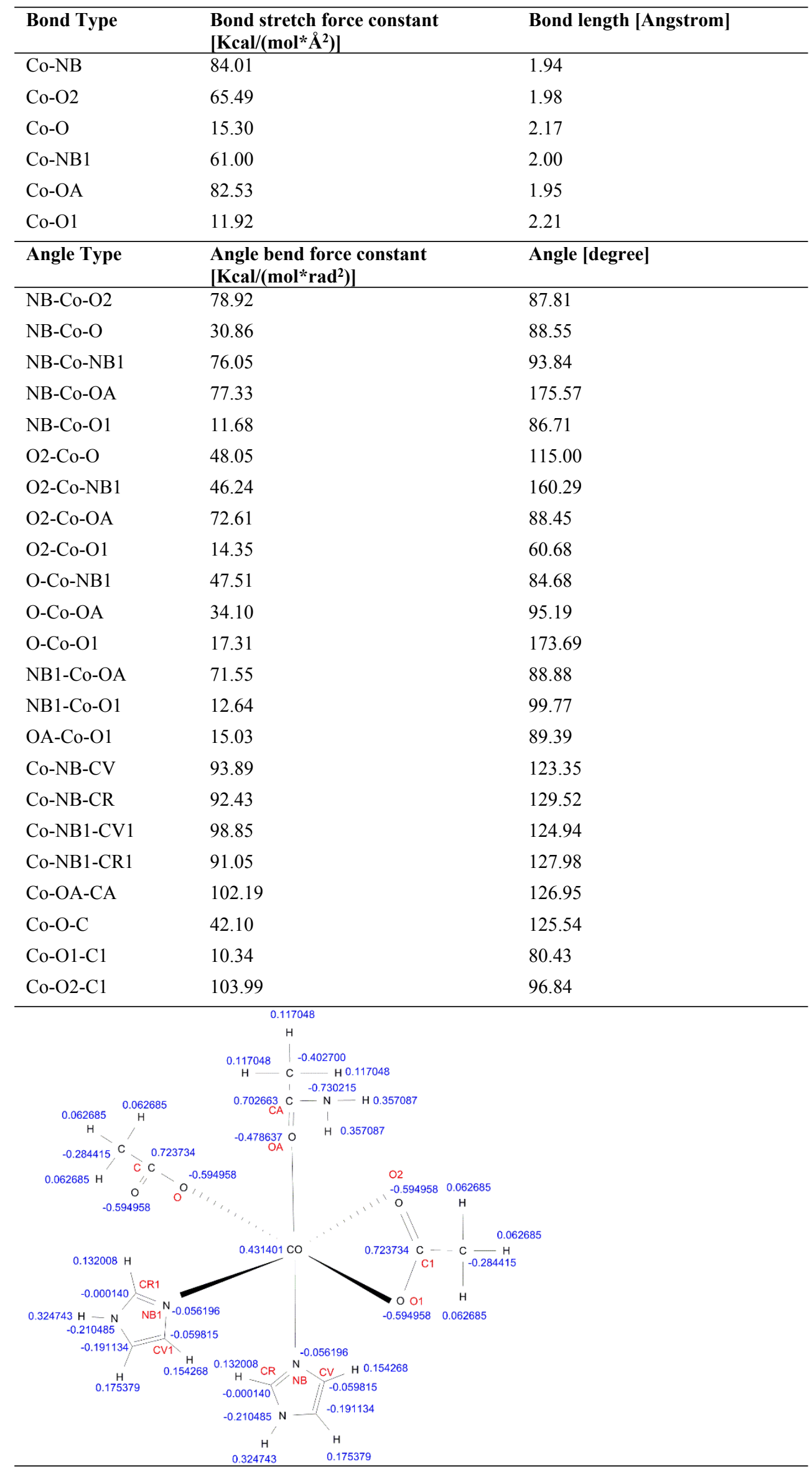


Table S5. The detailed structure and all bonding parameters of structure C5

\begin{tabular}{|c|c|c|}
\hline Bond Type & $\begin{array}{l}\text { Bond stretch force constant } \\
{\left[\mathrm{Kcal} /\left(\mathrm{mol}^{*} \AA^{2}\right)\right]}\end{array}$ & Bond length [Angstrom] \\
\hline Co-NB & 74.00 & 1.96 \\
\hline Co-NB1 & 62.46 & 1.99 \\
\hline $\mathrm{Co}-\mathrm{OP}$ & 107.22 & 1.91 \\
\hline Co-OW & 70.70 & 1.98 \\
\hline Co-O1 & 18.19 & 2.12 \\
\hline $\mathrm{Co}-\mathrm{O} 2$ & 24.34 & 2.08 \\
\hline Angle Type & $\begin{array}{l}\text { Angle bend force constant } \\
{\left[\mathrm{Kcal} /\left(\mathrm{mol}^{*} \mathbf{r a d}^{2}\right)\right]}\end{array}$ & Angle [degree] \\
\hline NB-Co-NB1 & 84.43 & 94.70 \\
\hline NB-Co-OP & 78.91 & 86.77 \\
\hline NB-Co-OW & 100.83 & 171.07 \\
\hline NB-Co-O1 & 13.24 & 95.63 \\
\hline NB-Co-O2 & 17.32 & 80.51 \\
\hline NB1-Co-OP & 67.45 & 173.60 \\
\hline NB1-Co-OW & 77.69 & 91.65 \\
\hline NB1-Co-O1 & 11.39 & 85.35 \\
\hline NB1-Co-O2 & 19.61 & 83.83 \\
\hline OP-Co-OW & 76.55 & 87.62 \\
\hline OP-Co-O1 & 15.19 & 88.30 \\
\hline OP-Co-O2 & 14.67 & 102.57 \\
\hline OW-Co-O1 & 10.10 & 91.14 \\
\hline OW-Co-O2 & 12.91 & 93.96 \\
\hline $\mathrm{O} 1-\mathrm{Co}-\mathrm{O} 2$ & 9.34 & 168.16 \\
\hline Co-NB-CV & 89.64 & 135.62 \\
\hline Co-NB-CR & 84.40 & 116.94 \\
\hline Co-NB1-CV1 & 87.89 & 126.26 \\
\hline Co-NB1-CR1 & 86.90 & 126.38 \\
\hline Co-OP-P & 68.95 & 124.61 \\
\hline Co-OW-HW & 40.73 & 99.92 \\
\hline $\mathrm{Co}-\mathrm{O} 1-\mathrm{C} 1$ & 17.36 & 118.71 \\
\hline $\mathrm{Co}-\mathrm{O} 2-\mathrm{C} 2$ & 14.91 & 141.61 \\
\hline . & 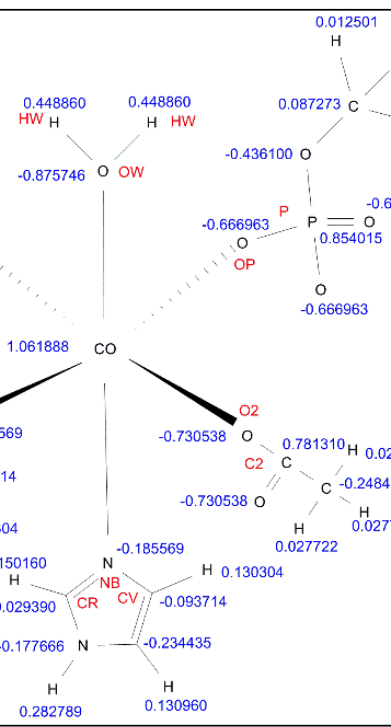 & \\
\hline
\end{tabular}


Table S6. The detailed structure and all bonding parameters of structure C6

\begin{tabular}{|c|c|c|}
\hline Bond Type & $\begin{array}{l}\text { Bond stretch force constant } \\
{\left[\mathrm{Kcal} /\left(\mathbf{m o l}^{*} \AA^{2}\right)\right]}\end{array}$ & Bond length [Angstrom] \\
\hline Co-NB & 41.3 & 1.99 \\
\hline Co-NB1 & 33.36 & 2.06 \\
\hline $\mathrm{Co}-\mathrm{O}$ & 77.81 & 1.97 \\
\hline Co-O1 & 104.16 & 1.89 \\
\hline $\mathrm{Co}-\mathrm{O} 2$ & 112.69 & 1.82 \\
\hline Co-OW & 78.35 & 1.96 \\
\hline Angle Type & $\begin{array}{l}\text { Angle bend force constant } \\
{\left[\mathrm{Kcal} /\left(\mathrm{mol}^{*} \mathrm{rad}^{2}\right)\right]}\end{array}$ & Angle [degree] \\
\hline NB-Co-NB1 & 27.76 & 99.71 \\
\hline NB-Co-O & 80.83 & 89.93 \\
\hline NB-Co-O1 & 60.01 & 123.76 \\
\hline NB-Co-O2 & 64.27 & 98.69 \\
\hline NB-Co-OW & 47.90 & 175.71 \\
\hline NB1-Co-O & 56.42 & 94.58 \\
\hline NB1-Co-O1 & 50.91 & 124.94 \\
\hline NB1-Co-O2 & 54.27 & 100.15 \\
\hline NB1-Co-OW & 43.68 & 80.51 \\
\hline O-Co-O1 & 63.37 & 115.34 \\
\hline $\mathrm{O}-\mathrm{Co}-\mathrm{O} 2$ & 51.51 & 161.36 \\
\hline O-Co-OW & 55.99 & 94.93 \\
\hline $\mathrm{O} 1-\mathrm{Co}-\mathrm{O} 2$ & 56.13 & 46.36 \\
\hline O1-Co-OW & 38.61 & 135.64 \\
\hline O2-Co-OW & 52.94 & 77.07 \\
\hline Co-NB-CV & 72.49 & 119.64 \\
\hline Co-NB-CR & 70.20 & 133.07 \\
\hline Co-NB1-CV1 & 38.96 & 136.92 \\
\hline Co-NB1-CR1 & 40.76 & 116.36 \\
\hline $\mathrm{Co}-\mathrm{O}-\mathrm{C}$ & 89.07 & 124.93 \\
\hline $\mathrm{Co}-\mathrm{O} 1-\mathrm{O} 2$ & 67.91 & 64.41 \\
\hline $\mathrm{Co}-\mathrm{O} 2-\mathrm{O} 1$ & 65.43 & 69.23 \\
\hline Co-OW-HW & 70.38 & 135.64 \\
\hline & 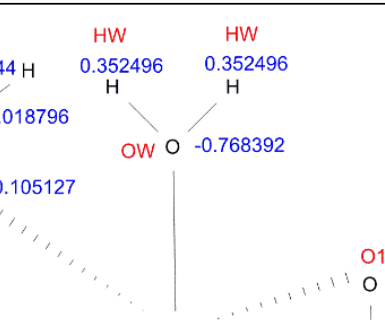 & \\
\hline $\begin{array}{r}0.126844 \mathrm{H} \\
-0.217885 \\
\mathrm{H}-\mathrm{N} \\
0.303099 \\
-0.224152 \\
\mathrm{H} \\
0.1503\end{array}$ & 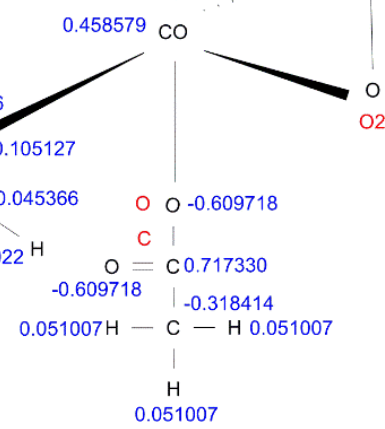 & \\
\hline
\end{tabular}


Table S7. The detailed structure and all bonding parameters of structure C7

\begin{tabular}{|c|c|c|}
\hline Bond Type & $\begin{array}{l}\text { Bond stretch force constant } \\
{\left[\mathrm{Kcal} /\left(\mathrm{mol}^{*} \AA^{2}\right)\right]}\end{array}$ & Bond length [Angstrom] \\
\hline Co-NB & 85.84 & 1.96 \\
\hline Co-NB1 & 84.26 & 1.95 \\
\hline Co-NB2 & 79.81 & 1.99 \\
\hline $\mathrm{Co}-\mathrm{O}$ & 87.88 & 1.94 \\
\hline $\mathrm{Co}-\mathrm{O} 1$ & 65.73 & 1.98 \\
\hline $\mathrm{Co}-\mathrm{O} 2$ & 108.97 & 1.91 \\
\hline Angle Type & $\begin{array}{l}\text { Angle bend force constant } \\
{\left[\mathrm{Kcal} /\left(\mathrm{mol}^{*} \mathrm{rad}^{2}\right)\right]}\end{array}$ & Angle [degree] \\
\hline NB-Co-NB1 & 97.44 & 94.51 \\
\hline NB-Co-NB2 & 84.42 & 93.35 \\
\hline $\mathrm{NB}-\mathrm{Co}-\mathrm{O}$ & 69.01 & 85.46 \\
\hline NB-Co-O1 & 63.18 & 171.28 \\
\hline NB-Co-O2 & 97.33 & 89.83 \\
\hline NB1-Co-NB2 & 80.01 & 95.56 \\
\hline NB1-Co-O & 66.67 & 88.07 \\
\hline NB1-Co-O1 & 53.37 & 91.97 \\
\hline NB1-Co-O2 & 88.14 & 173.65 \\
\hline NB2-Co-O & 63.79 & 176.27 \\
\hline NB2-Co-O1 & 48.80 & 91.82 \\
\hline NB2-Co-O2 & 75.03 & 88.80 \\
\hline $\mathrm{O}-\mathrm{Co}-\mathrm{O} 1$ & 72.12 & 88.93 \\
\hline $\mathrm{O}-\mathrm{Co}-\mathrm{O} 2$ & 80.09 & 87.66 \\
\hline $\mathrm{O} 1-\mathrm{Co}-\mathrm{O} 2$ & 69.82 & 83.25 \\
\hline Co-NB-CV & 98.82 & 130.03 \\
\hline Co-NB-CR & 93.34 & 122.79 \\
\hline Co-NB1-CV1 & 103.97 & 126.15 \\
\hline Co-NB1-CR1 & 99.98 & 127.28 \\
\hline $\mathrm{Co}-\mathrm{NB} 2-\mathrm{CV} 2$ & 83.3 & 133.48 \\
\hline Co-NB2-CR2 & 80.85 & 120.46 \\
\hline $\mathrm{Co}-\mathrm{O}-\mathrm{C}$ & 70.94 & 136.07 \\
\hline Co-O1-C1 & 50.36 & 112.27 \\
\hline $\mathrm{Co}-\mathrm{O} 2-\mathrm{C} 2$ & 105.99 & 115.63 \\
\hline 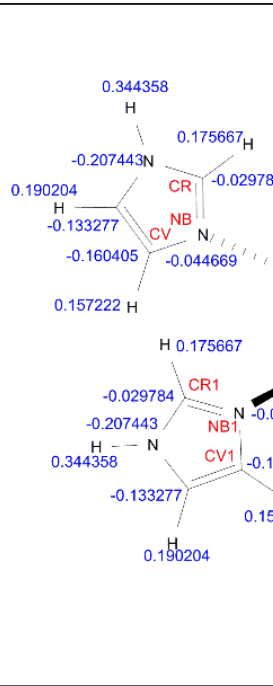 & 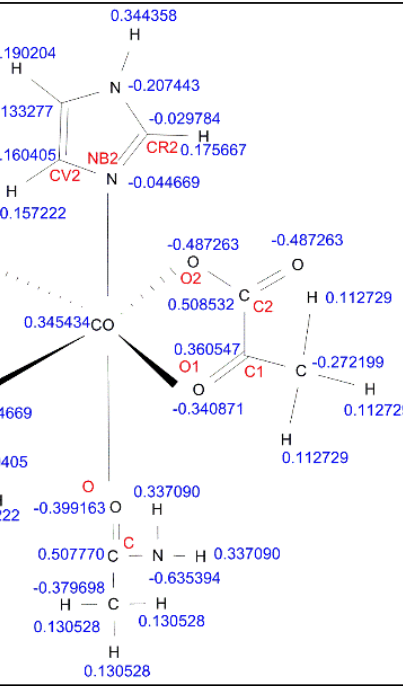 & \\
\hline
\end{tabular}


Table S8. The detailed structure and all bonding parameters of structure C8

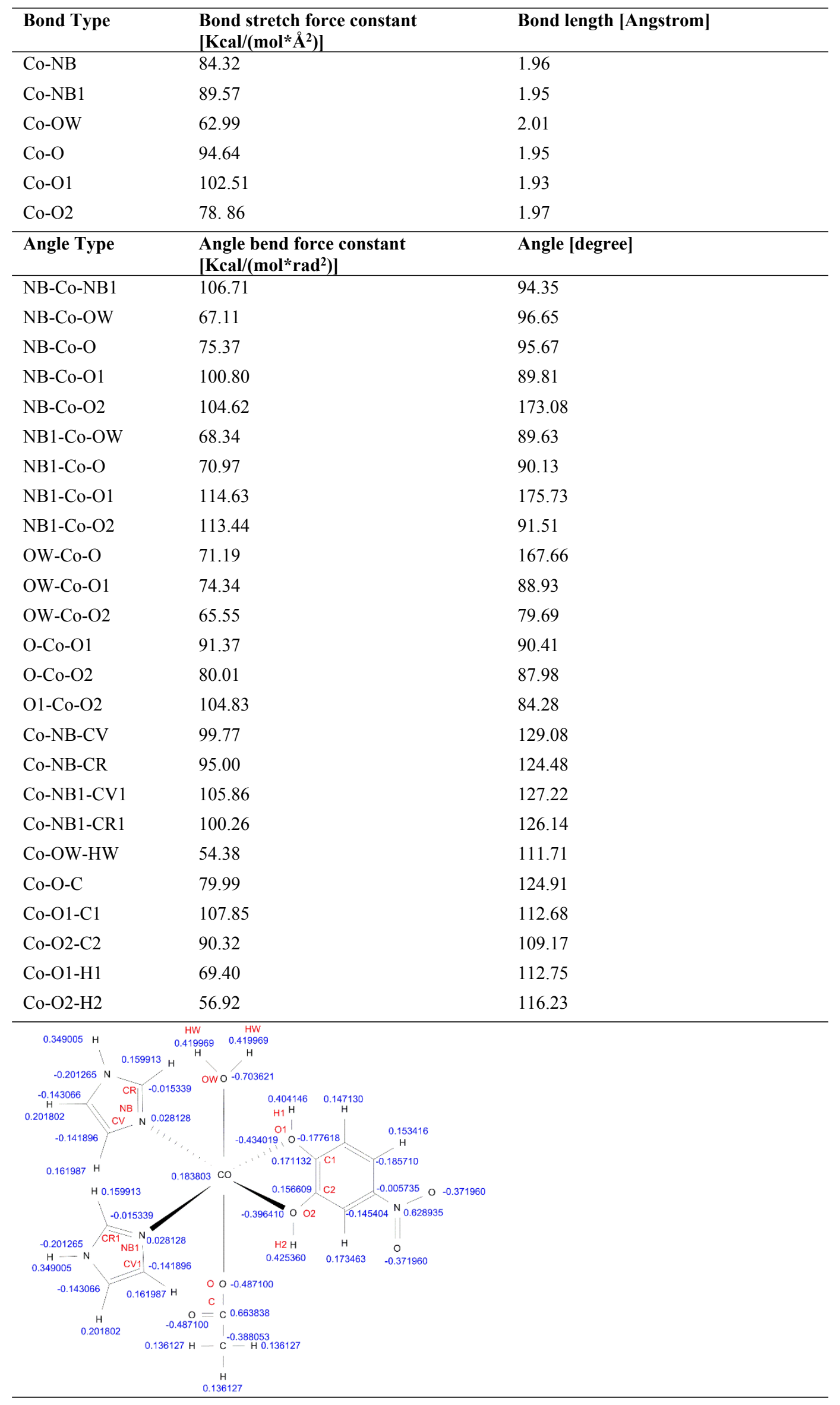


Table S9. The detailed structure and all bonding parameters of structure C9

\begin{tabular}{|c|c|c|}
\hline Bond Type & $\begin{array}{l}\text { Bond stretch force constant } \\
{\left[\mathrm{Kcal} /\left(\mathrm{mol}^{*} \AA^{2}\right)\right]}\end{array}$ & Bond length [Angstrom] \\
\hline Co-NB & 82.49 & 1.96 \\
\hline Co-NB1 & 89.41 & 1.95 \\
\hline $\mathrm{Co}-\mathrm{O}$ & 16.33 & 2.09 \\
\hline $\mathrm{Co}-\mathrm{N}$ & 78.21 & 1.98 \\
\hline $\mathrm{Co}-\mathrm{O} 1$ & 99.84 & 1.93 \\
\hline $\mathrm{Co}-\mathrm{O} 2$ & 9.70 & 2.19 \\
\hline Angle Type & $\begin{array}{l}\text { Angle bend force constant } \\
{\left[\mathrm{Kcal} /\left(\mathrm{mol}^{*} \mathbf{r a d}^{2}\right)\right]}\end{array}$ & Angle [degree] \\
\hline NB-Co-NB1 & 94.69 & 93.27 \\
\hline NB-Co-O & 12.63 & 93.19 \\
\hline NB-Co-N & 112.24 & 172.75 \\
\hline NB-Co-O1 & 92.60 & 89.08 \\
\hline NB-Co-O2 & 7.99 & 97.41 \\
\hline NB1-Co-O & 11.35 & 93.57 \\
\hline NB1-Co-N & 81.52 & 92.27 \\
\hline NB1-Co-O1 & 83.90 & 172.98 \\
\hline NB1-Co-O2 & 9.25 & 98.03 \\
\hline $\mathrm{O}-\mathrm{Co}-\mathrm{N}$ & 13.99 & 91.136 \\
\hline O-Co-O1 & 10.93 & 92.90 \\
\hline $\mathrm{O}-\mathrm{Co}-\mathrm{O} 2$ & 7.69 & 163.78 \\
\hline N-Co-O1 & 86.10 & 84.88 \\
\hline $\mathrm{N}-\mathrm{Co}-\mathrm{O} 2$ & 10.12 & 77.17 \\
\hline $\mathrm{O} 1-\mathrm{Co}-\mathrm{O} 2$ & 13.67 & 75.09 \\
\hline Co-NB-CV & 98.34 & 125.58 \\
\hline Co-NB-CR & 95.42 & 128.13 \\
\hline Co-NB1-CV1 & 77.96 & 127.78 \\
\hline Co-NB1-CR1 & 77.30 & 125.41 \\
\hline Co-O1-H1 & 89.43 & 78.110 \\
\hline $\mathrm{Co}-\mathrm{O} 2-\mathrm{H} 2$ & 12.46 & 93.26 \\
\hline Co-O-C & 14.93 & 121.898 \\
\hline $\mathrm{Co}-\mathrm{N}-\mathrm{HN}$ & 46.72 & 115.1 \\
\hline $\mathrm{Co}-\mathrm{N}-\mathrm{C} 3$ & 102.10 & 104.39 \\
\hline $\mathrm{Co}-\mathrm{O} 1-\mathrm{C} 1$ & 126.34 & 112.33 \\
\hline $\mathrm{Co}-\mathrm{O} 2-\mathrm{C} 2$ & 13.69 & 101.296 \\
\hline (196761 & (161728 & \\
\hline
\end{tabular}


Table S10. The detailed structure and all bonding parameters of structure $\mathrm{C} 10$

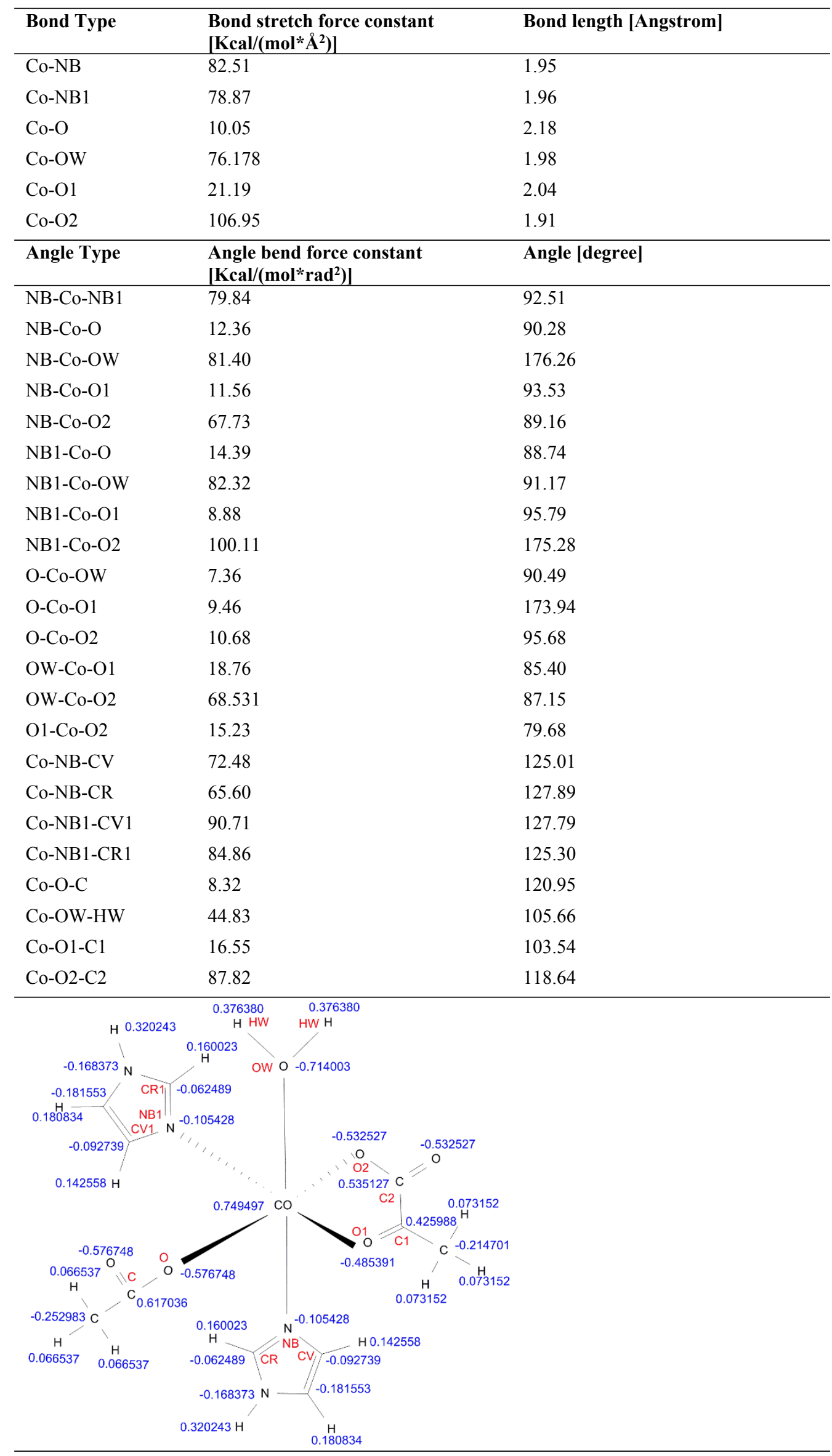


Table S11. The detailed structure and all bonding parameters of structure C11

\begin{tabular}{|c|c|c|}
\hline Bond Type & $\begin{array}{l}\text { Bond stretch force constant } \\
{\left[\mathrm{Kcal} /\left(\mathrm{mol}^{*} \AA^{2}\right)\right]}\end{array}$ & Bond length [Angstrom] \\
\hline Co-S & 42.43 & 2.37 \\
\hline Co-NB & 81.84 & 1.98 \\
\hline Co-NB1 & 73.99 & 1.99 \\
\hline $\mathrm{Co}-\mathrm{O}$ & 75.60 & 1.96 \\
\hline $\mathrm{Co}-\mathrm{OH}$ & 51.83 & 2.25 \\
\hline Angle Type & $\begin{array}{l}\text { Angle bend force constant } \\
{\left[\mathrm{Kcal} /\left(\mathrm{mol}^{*} \mathbf{r a d}^{2}\right)\right]}\end{array}$ & Angle [degree] \\
\hline S-Co-NB & 82.90 & 90.82 \\
\hline S-Co-NB1 & 66.82 & 98.23 \\
\hline S-Co-O & 55.16 & 162.13 \\
\hline S-Co-OH & 17.53 & 94.39 \\
\hline NB-Co-NB1 & 14.82 & 128.79 \\
\hline NB-Co-O & 80.02 & 90.64 \\
\hline NB-Co-OH & 12.96 & 138.35 \\
\hline NB1-Co-O & 54.85 & 94.71 \\
\hline NB1-Co-OH & 16.09 & 91.29 \\
\hline $\mathrm{O}-\mathrm{Co}-\mathrm{OH}$ & 10.34 & 73.02 \\
\hline Co-S-CS & 111.78 & 106.92 \\
\hline Co-S-HS & 41.59 & 106.91 \\
\hline Co-NB-CV & 71.12 & 125.83 \\
\hline Co-NB-CR & 65.33 & 127.57 \\
\hline Co-NB1-CV1 & 71.96 & 122.07 \\
\hline Co-NB1-CR1 & 70.18 & 131.13 \\
\hline $\mathrm{Co}-\mathrm{O}-\mathrm{C}$ & 68.53 & 122.12 \\
\hline $\mathrm{Co}-\mathrm{OH}-\mathrm{N}$ & 16.39 & 105.99 \\
\hline $\mathrm{Co}-\mathrm{OH}-\mathrm{HO}$ & 19.33 & 111.47 \\
\hline $\begin{array}{r}0.16 \\
0.089111 \\
H \\
0.013047 ! \\
0.089111 \mathrm{H}-\mathrm{C} \\
0.089111 \mathrm{H}\end{array}$ & 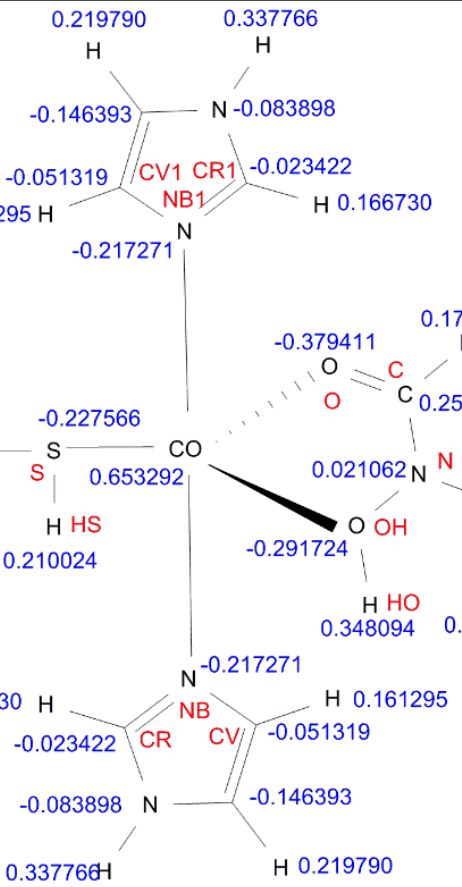 & $\begin{array}{l}.159674 \\
6274 \\
674\end{array}$ \\
\hline
\end{tabular}


Table S12. The detailed structure and all bonding parameters of structure C12

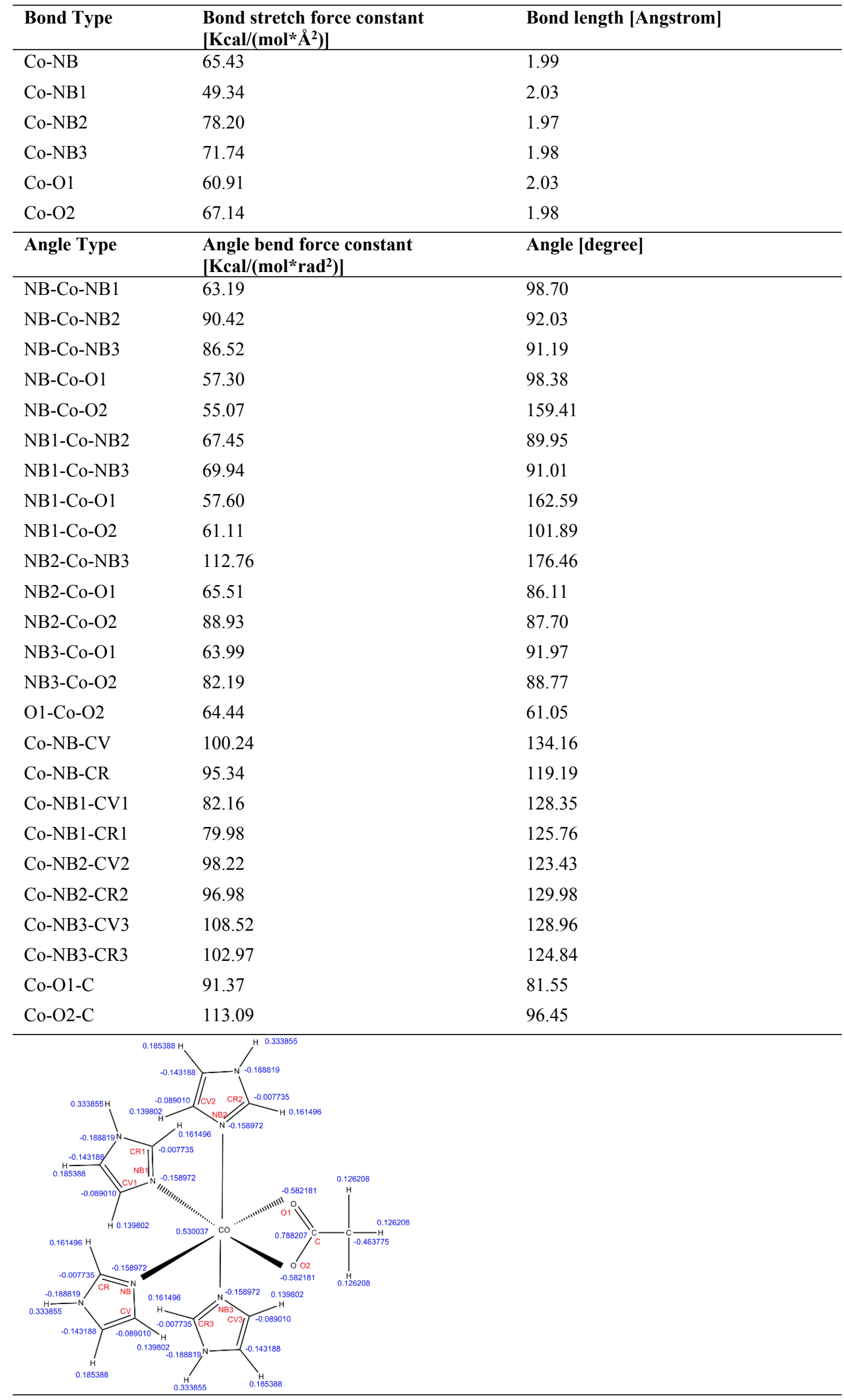


Table S13. The detailed structure and all bonding parameters of structure C13

\begin{tabular}{|c|c|c|}
\hline Bond Type & $\begin{array}{l}\text { Bond stretch force constant } \\
{\left[\mathrm{Kcal} /\left(\mathrm{mol}^{*} \AA^{2}\right)\right]}\end{array}$ & Bond length [Angstrom] \\
\hline Co-NB & 75.63 & 1.98 \\
\hline Co-NB1 & 86.45 & 1.94 \\
\hline $\mathrm{Co}-\mathrm{O}$ & 85.58 & 1.95 \\
\hline Co-O1 & 137.46 & 1.87 \\
\hline $\mathrm{Co}-\mathrm{O} 2$ & 79.00 & 1.97 \\
\hline Angle Type & $\begin{array}{l}\text { Angle bend force constant } \\
{\left[\mathrm{Kcal} /\left(\mathrm{mol}^{*} \mathbf{r a d}^{2}\right)\right]}\end{array}$ & Angle [degree] \\
\hline NB-Co-NB1 & 74.91 & 82.62 \\
\hline NB-Co-O & 79.11 & 82.03 \\
\hline NB-Co-O1 & 83.79 & 83.19 \\
\hline NB-Co-O2 & 73.77 & 102.39 \\
\hline NB1-Co-O & 102.60 & 91.59 \\
\hline NB1-Co-O1 & 43.59 & 164.98 \\
\hline NB1-Co-O2 & 90.23 & 92.18 \\
\hline $\mathrm{O}-\mathrm{Co}-\mathrm{O} 1$ & 89.72 & 91.46 \\
\hline $\mathrm{O}-\mathrm{Co}-\mathrm{O} 2$ & 74.86 & 174.50 \\
\hline $\mathrm{O} 1-\mathrm{Co}-\mathrm{O} 2$ & 89.83 & 85.92 \\
\hline Co-NB-CV & 92.67 & 127.17 \\
\hline Co-NB-CR & 93.10 & 126.07 \\
\hline Co-NB1-CV1 & 94.29 & 126.73 \\
\hline Co-NB1-CR1 & 94.13 & 126.64 \\
\hline $\mathrm{Co}-\mathrm{O}-\mathrm{C}$ & 100.42 & 95.00 \\
\hline $\mathrm{Co}-\mathrm{O} 1-\mathrm{C} 1$ & 120.62 & 117.20 \\
\hline $\mathrm{Co}-\mathrm{O} 2-\mathrm{C} 2$ & 106.43 & 108.85 \\
\hline $\mathrm{Co}-\mathrm{O} 2-\mathrm{H} 2$ & 42.95 & 108.04 \\
\hline -0. & 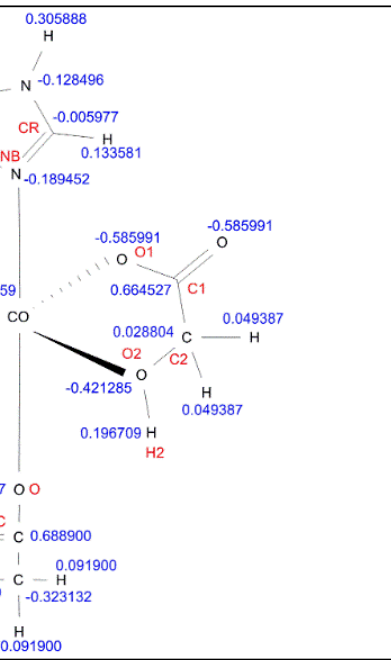 & \\
\hline
\end{tabular}


Table S14. The detailed structure and all bonding parameters of structure C14

\begin{tabular}{|c|c|c|}
\hline Bond Type & $\begin{array}{l}\text { Bond stretch force constant } \\
\left.\left[\text { Kcal/(mol* } \AA^{2}\right)\right]\end{array}$ & Bond length [Angstrom] \\
\hline Co-NB & 79.31 & 1.96 \\
\hline Co-NB1 & 68.61 & 1.98 \\
\hline $\mathrm{Co}-\mathrm{O}$ & 74.58 & 1.94 \\
\hline $\mathrm{Co}-\mathrm{O} 1$ & 45.92 & 2.08 \\
\hline $\mathrm{Co}-\mathrm{OP}$ & 115.76 & 1.88 \\
\hline Angle Type & $\begin{array}{l}\text { Angle bend force constant } \\
{\left[\mathrm{Kcal} /\left(\mathrm{mol}^{*} \mathrm{rad}^{2}\right)\right]}\end{array}$ & Angle [degree] \\
\hline NB-Co-NB1 & 122.38 & 176.65 \\
\hline NB-Co-O & 87.14 & 94.51 \\
\hline NB-Co-O1 & 42.49 & 92.29 \\
\hline NB-Co-OP & 48.00 & 89.46 \\
\hline NB1-Co-O & 91.28 & 87.38 \\
\hline NB1-Co-O1 & 40.37 & 89.52 \\
\hline NB1-Co-OP & 51.79 & 87.61 \\
\hline O-Co-O1 & 37.90 & 112.53 \\
\hline $\mathrm{O}-\mathrm{Co}-\mathrm{OP}$ & 64.66 & 153.14 \\
\hline $\mathrm{O} 1-\mathrm{Co}-\mathrm{OP}$ & 55.01 & 93.79 \\
\hline Co-NB-CV & 107.60 & 129.37 \\
\hline Co-NB-CR & 100.17 & 123.50 \\
\hline Co-NB1-CV & 98.87 & 126.90 \\
\hline Co-NB1-CR & 97.05 & 126.21 \\
\hline $\mathrm{Co}-\mathrm{O}-\mathrm{C}$ & 68.52 & 137.77 \\
\hline $\mathrm{Co}-\mathrm{O} 1-\mathrm{C} 1$ & 36.10 & 111.80 \\
\hline Co-O1-H1 & 30.46 & 99.87 \\
\hline Co-OP-P & 56.89 & 136.11 \\
\hline $\begin{array}{r}0.017630 \\
H-C \\
-0.114029 \mid \\
H \\
0.0176\end{array}$ & 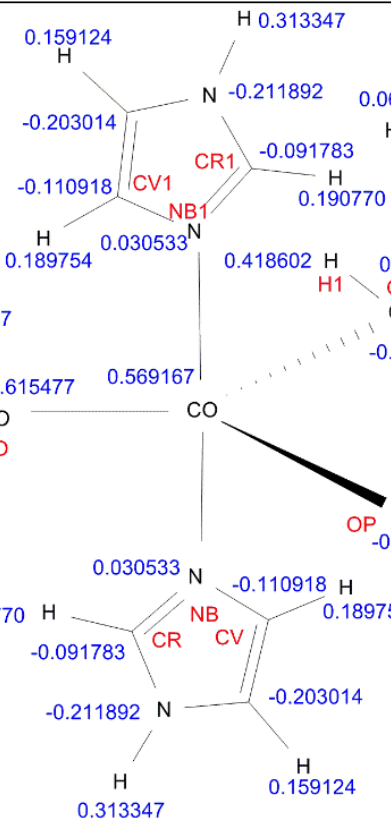 & $\begin{array}{l}.067331 \\
\mathrm{H} \\
0481 \\
109050 \\
\mathrm{H} \\
0.415827 \\
-0.618610 \\
-0 \\
\\
18610\end{array}$ \\
\hline
\end{tabular}


Table S15. The detailed structure and all bonding parameters of structure C15

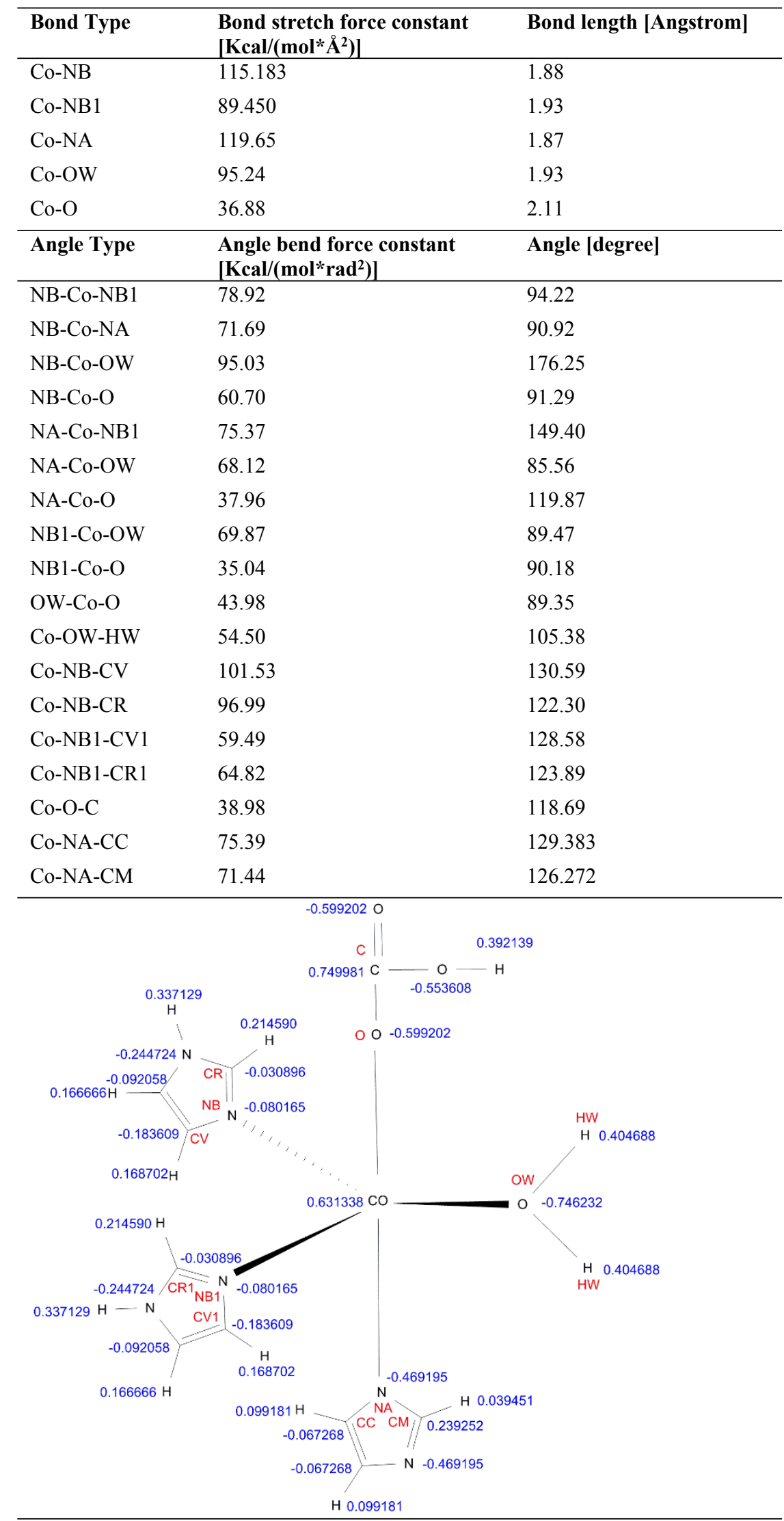


Table S16. The detailed structure and all bonding parameters of structure C16

\begin{tabular}{|c|c|c|}
\hline Bond Type & $\begin{array}{l}\text { Bond stretch force constant } \\
{\left[\mathrm{Kcal} /\left(\mathrm{mol}^{*} \AA^{2}\right)\right]}\end{array}$ & Bond length [Angstrom] \\
\hline Co-NB & 63.68 & 1.97 \\
\hline Co-NB1 & 55.79 & 2.05 \\
\hline $\mathrm{Co}-\mathrm{O} 1$ & 94.65 & 1.93 \\
\hline Co-OP1 & 114.01 & 1.91 \\
\hline Co-OP2 & 67.69 & 1.98 \\
\hline Angle Type & $\begin{array}{l}\text { Angle bend force constant } \\
{\left[\mathrm{Kcal} /\left(\mathrm{mol}^{*} \mathrm{rad}^{2}\right)\right]}\end{array}$ & Angle [degree] \\
\hline NB-Co-NB1 & 55.21 & 92.84 \\
\hline NB-Co-O1 & 86.07 & 93.14 \\
\hline NB-Co-OP1 & 61.48 & 166.41 \\
\hline NB-Co-OP2 & 89.09 & 95.54 \\
\hline NB1-Co-O1 & 49.50 & 90.08 \\
\hline NB1-Co-OP1 & 55.13 & 98.95 \\
\hline NB1-Co-OP2 & 52.39 & 100.94 \\
\hline O1-Co-OP1 & 80.70 & 93.58 \\
\hline O1-Co-OP2 & 81.85 & 165.58 \\
\hline OP1-Co-OP2 & 89.04 & 75.67 \\
\hline Co-NB-CV & 95.85 & 126.24 \\
\hline Co-NB-CR & 93.55 & 126.91 \\
\hline Co-NB1-CV1 & 56.86 & 129.65 \\
\hline Co-NB1-CR1 & 53.71 & 126.06 \\
\hline Co-O1-C & 92.08 & 116.78 \\
\hline Co-OP1-P & 124.13 & 102.68 \\
\hline Co-OP2-P & 105.23 & 91.60 \\
\hline $\mathrm{Co}-\mathrm{OP} 2-\mathrm{C} 1$ & 91.03 & 129.69 \\
\hline 0.0 .08 & (281 & - \\
\hline
\end{tabular}




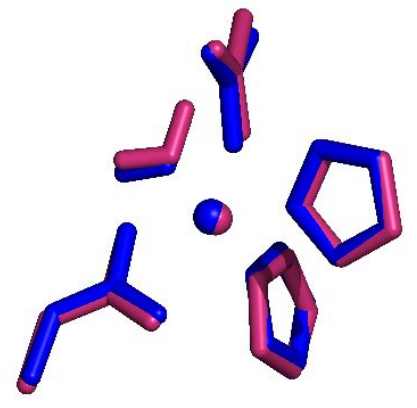

Bonded model with RESP charge

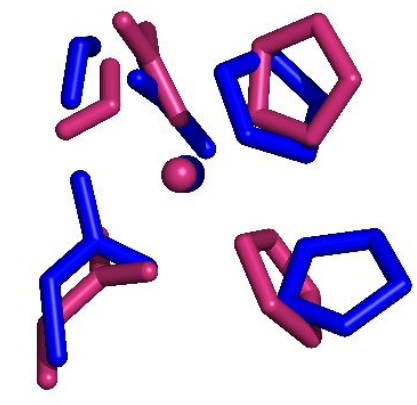

Nonbonded model with RESP charge
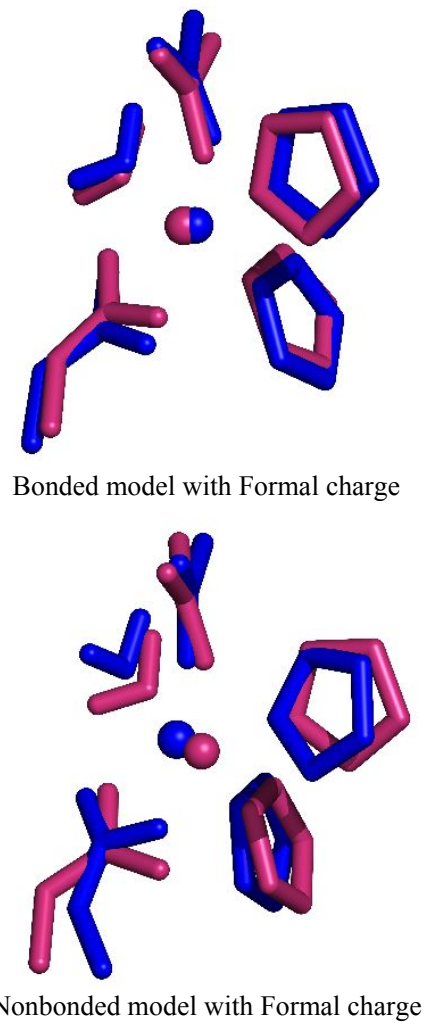

Figure S1. The three-dimensional structures of FFM-based minimized structures (blue) versus the QM optimized structures (purple) for model C2 


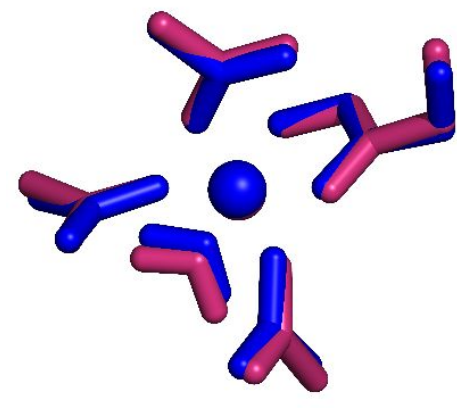

Bonded model with RESP charge

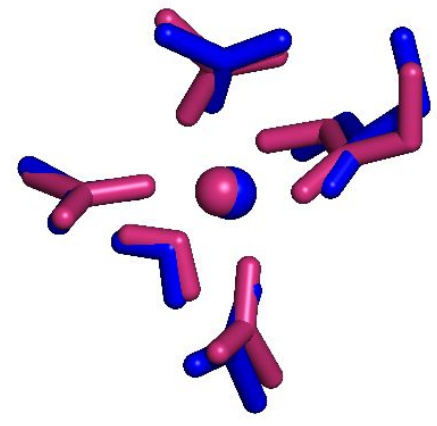

Nonbonded model with RESP charge

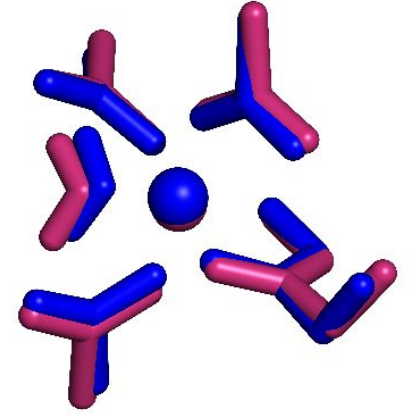

Bonded model with Formal charge

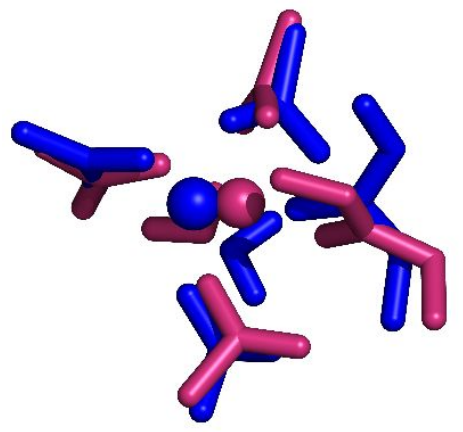

Nonbonded model with Formal charge

Figure S2. The three-dimensional structures of FFM-based minimized structures (blue) versus the QM optimized structures (purple) for model C3 


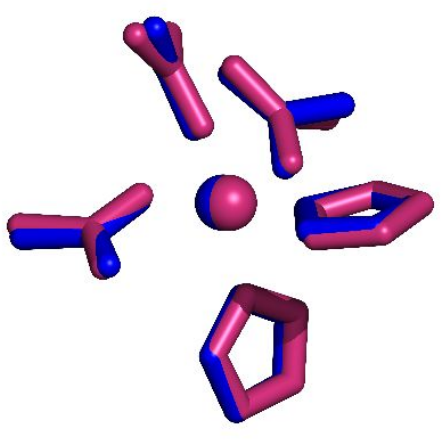

Bonded model with RESP charge

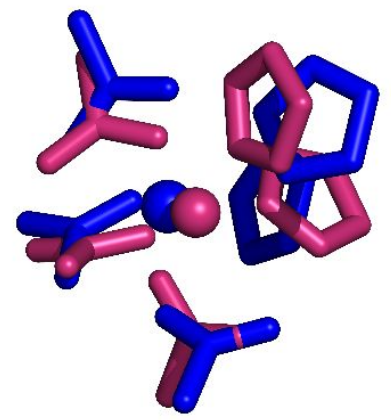

Nonbonded model with RESP charge

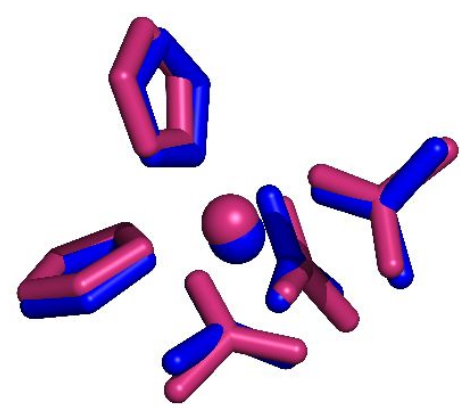

Bonded model with Formal charge

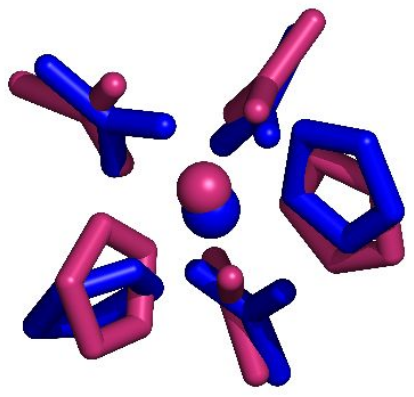

Nonbonded model with Formal charge

Figure S3. The three-dimensional structures of FFM-based minimized structures (blue) versus the QM optimized structures (purple) for model C4 


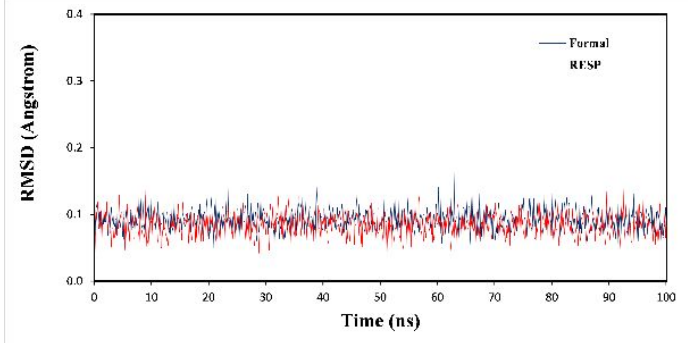

Bonded model in vacuum

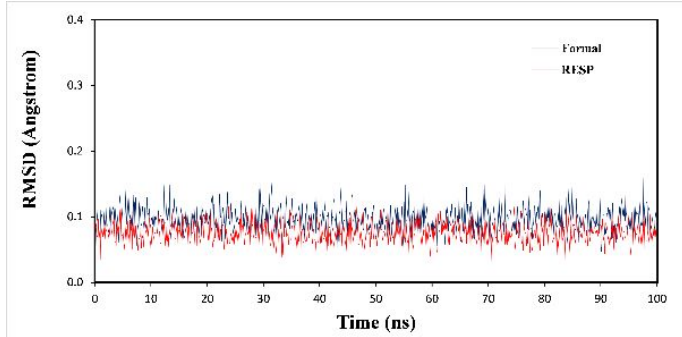

Bonded model in water

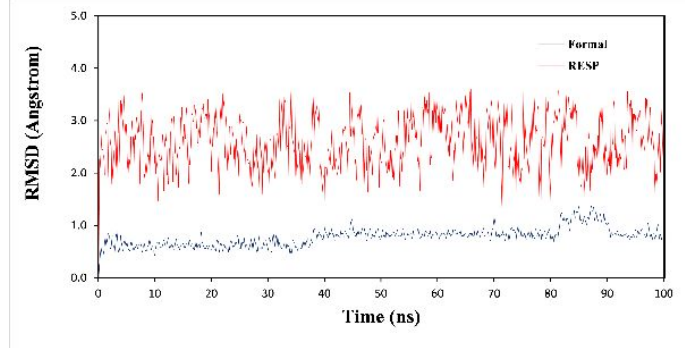

Nonbonded model in vacuum

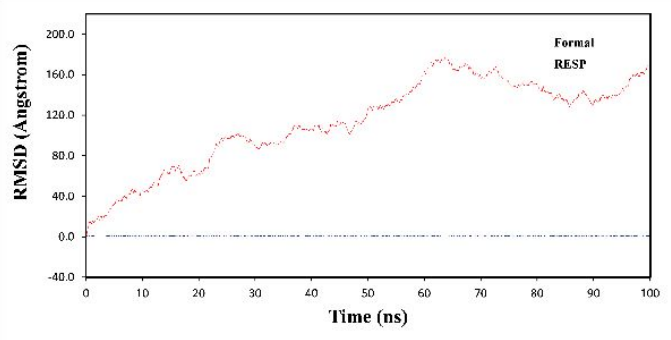

Nonbonded model in water

Figure S4. The RMSD values (obtained from the MD simulations) for C2 model in water and vacuum 


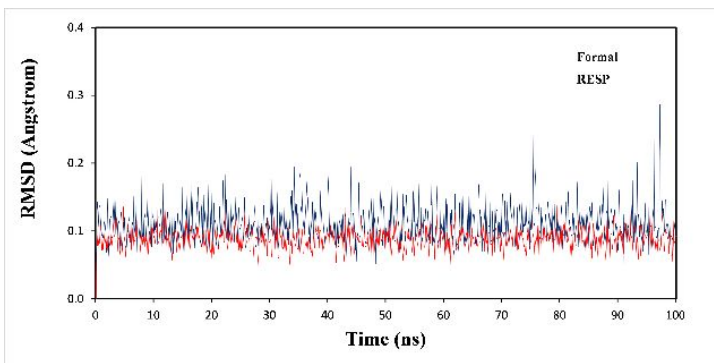

Bonded model in vacuum

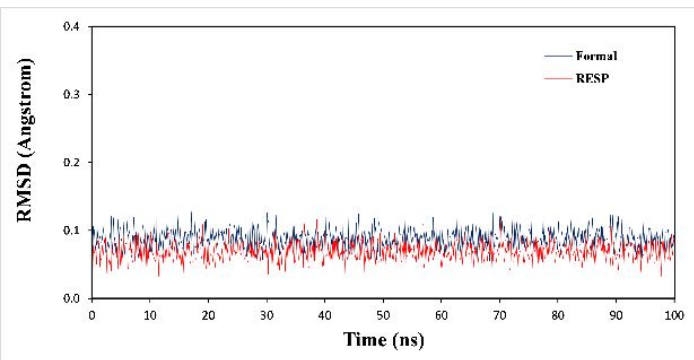

Bonded model in water

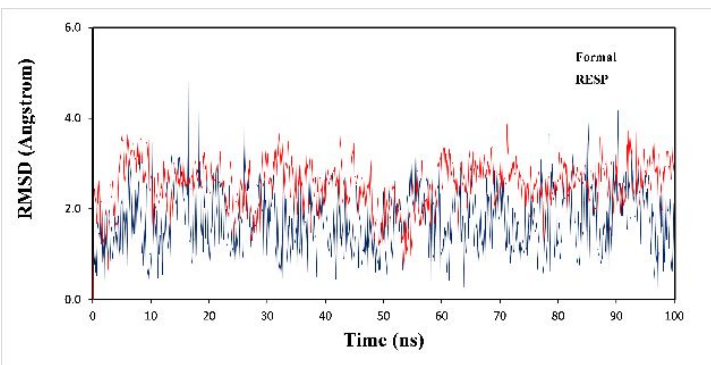

Nonbonded model in vacuum

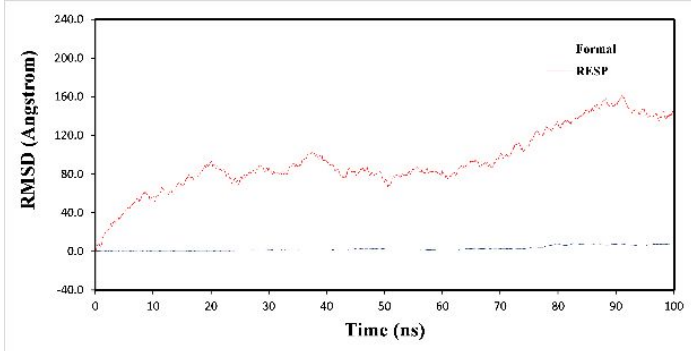

Nonbonded model in water

Figure S5. The RMSD values (obtained from the MD simulations) for C3 model in water and vacuum 


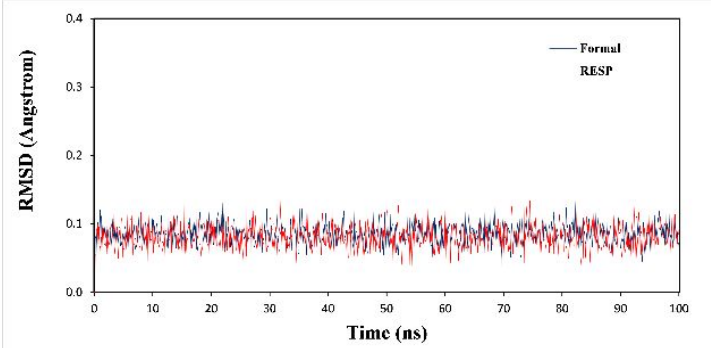

Bonded model in vacuum

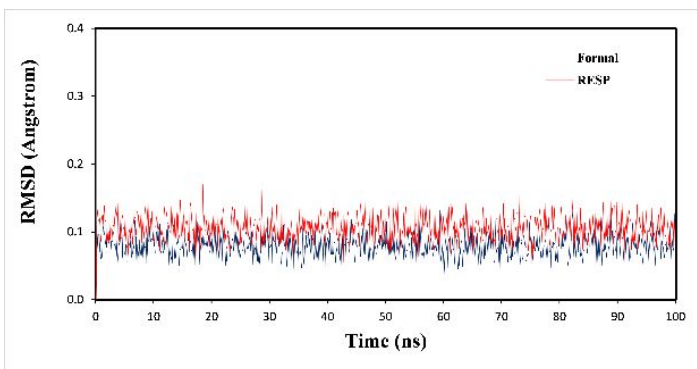

Bonded model in water

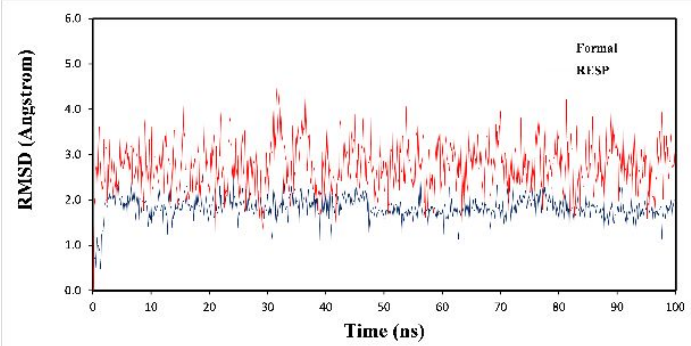

Nonbonded model in vacuum

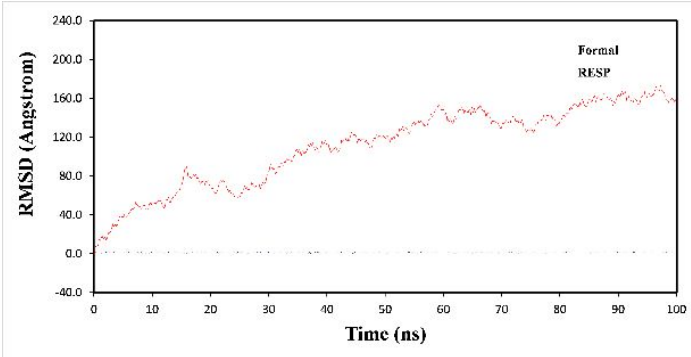

Nonbonded model in water

Figure S6. The RMSD values (obtained from the MD simulations) for C4 model in water and vacuum 